\title{
René Magritte, illustrateur du cours de Linguistique générale de Ferdinand de Saussure ${ }^{1}$, (extrait).
}

Jean David

\section{LES MOTS ET LES IMAGES}

Un objet ne thent pas trllement $\lambda$ son tou qu'on ae puise lai ta troever un sutse qui lấ convieone mieux

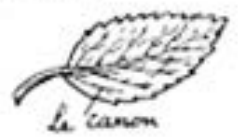

If y a des objets qu $x$ passat de nom :

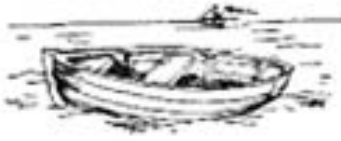

Vo toot he sert parfois qu'd se Alsignet ఢ̣i-maème :

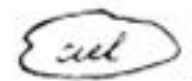

Lo objet rencontre son image, un objet reacoetre son som. II arrive que Timage et le hein de cet objet se rencoatrest

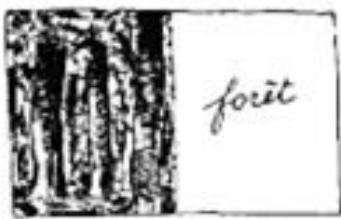

Parfois le nom d'un objet tient lies d'une imser

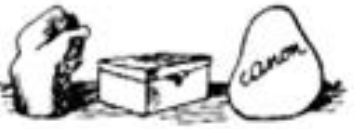

Un mot perut preadre ta place diun ebjet dans la realite:

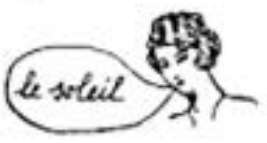

Use image peut prebolte h piace d'un mot dass une proposition.

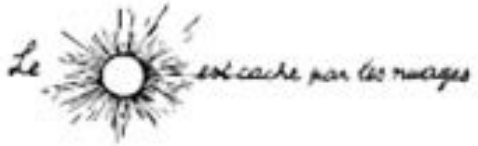

Uis objet fait supposec ga'ti v rn a d'autres derntre lui :



Toat lend a lare penser quil y a peu de relatios enter so objet et se qui le represeate

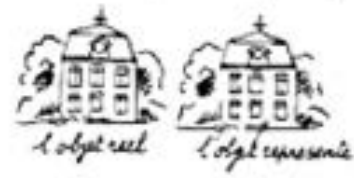

Les mots qui servent a desigar deux objet. ciflefrets ar mostrest pas ce cur ptut utpare ers objets tun de lavite

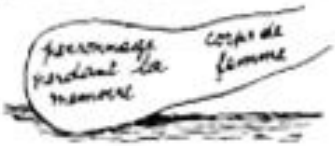

Duns en tabdrau, les mots vont de ta méme substance que les images

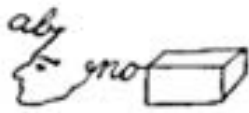

Oa voit autrement les images et les Enots dans ua tablezu

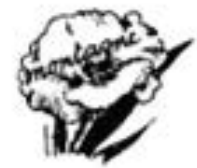

Une forme qutkençae prut remplact: rimage d'ua objet

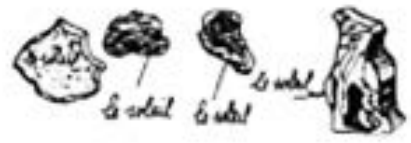

Un obyet he fait jamass ke mithe effien çer soh bom oy que wee imagr

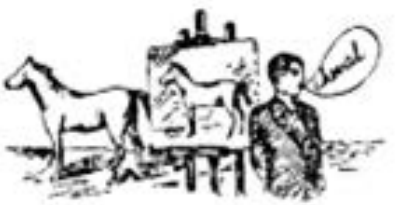

Or. les senteun visibles des objets, dars ta realiue, se toschent cotnror ist formaient une prosalqzer :

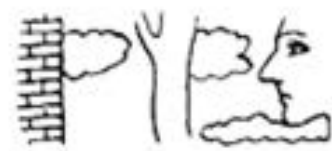

Let figutes vagues ont une signification agisa necresaire auss parfaite que les precises

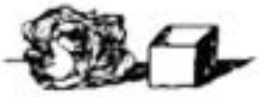

Parieis, les noms ecrits tans on tablesu designest des chosen presces, it les imazes tect shours vagues



Ou bien le contraite :

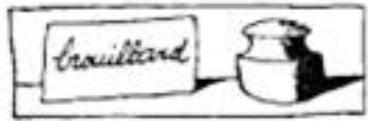

René Mavertre.

1 M. Jean DAVID. http://documents.irevues.inist.fr/bitstream/handle/2042/34531/ANM_2008_125.pdf?seq 
Précisons tout de suite le sens du titre de cette communication. Les seules illustrations que contiennent les éditions successives du Cours de linguistique générale -au catalogue de Payot depuis 1916- sont quelques croquis rudimentaires, utilisés par exemple pour donner à voir la structure complexe du signe.

Magritte n'a pas illustré Saussure comme Gustave Doré a illustré La Fontaine ou Victor Hugo. Ce que je me propose de faire en donnant ce titre à ma communication, c'est de montrer que certains dessins et tableaux de Magritte sont des traductions picturales de quelques notions fondamentales du Cours de Saussure ou tout au moins d'implications de celles-ci. A ce titre, certes, ils pourraient être utilisés, une fois affublés d'un commentaire de linguiste, pour mettre une note de couleur et d'humour dans l'austère traité du professeur genevois, mais ceci n'a pas encore été fait.

Ferdinand de Saussure et René Magritte ne se sont évidemment jamais rencontrés. Magritte avait quinze ans en 1913, année de la mort de Saussure. Pour ce qui est du Cours, publié trois ans après la mort du linguiste, Magritte, qui a dit de lui-même, "Je ne suis pas un peintre, je suis un homme qui pense», aurait sans doute été à même de le lire, mais rien dans sa volumineuse correspondance ni dans les témoignages de son entourage ne donne à penser qu'il l'ait fait. Ceci légitime la question de savoir par quel cheminement les idées du linguiste sont venues prendre forme sous le pinceau du peintre.
Cette question se pose pour bien d'autres cas de proximité évidente. Elle reste parfois sans réponse. J'ai eu à m'intéresser de très près à la parenté manifeste de la structure de la phrase telle que Lucien Tesnière la décrit dans ses Eléments de linguistique structurale et de la fonction propositionnelle telle que la conçoit le logicien allemand Frege. Jean Fourquet, qui a édité ces Eléments de syntaxe structurale, ouvrage posthume comme l'est le Cours de linguistique générale, avait eu avec Lucien Tesnière d'interminables conversations pendant les années de l'immédiat avant-guerre où s'élaboraient ces Eléments. Il m' a assuré que jamais Tesnière n'avait fait allusion à des travaux de logiciens modernes. La parenté est là, inexpliquée. Faute de mieux, on dira que la convergence était dans l'air du temps...

Dans le cas de la relation des idées de Saussure et des œuvres graphiques de Magritte, on peut aller un tout petit peu plus avant dans l'explication. Très jeune, Magritte a fréquenté les milieux dadaïstes puis surréalistes, à Bruxelles et à Paris. Il a d'ailleurs été relevé que Magritte fréquentait plus volontiers les artisans du verbe que les manieurs de pinceaux, les poètes plutôt que les peintres. Dans les orientations littéraires de ces mouvements, la réflexion sur le langage, le jeu avec le langage, de l'écriture automatique au cadavre exquis, tiennent une place centrale. Et l'on peut montrer que dans la réflexion, les idées de Saussure ont leur place. 